VoL. 75 (2007) [17-22]

\title{
INVERSE LIMITS AS LIMITS WITH RESPECT TO THE HAUSDORFF METRIC
}

\author{
IzTOK BANIČ
}

\begin{abstract}
We show that the inverse limit of any inverse sequence of compact metric spaces and surjective bonding maps is in fact the limit of a sequence of homeomorphic copies of the same spaces with respect to the Hausdorff metric.
\end{abstract}

\section{INTRODUCTION}

Inverse limits of inverse sequences of compact metric spaces have been the subject of research for many years. As very complicated spaces may be presented as inverse limits of simple spaces and simple bonding maps, a representation of spaces as inverse limits can be very useful when studying their properties. For examples see $[\mathbf{8}$, p. 205], $[4,5]$.

It is well known that the limits of the sequences of compact metric spaces with respect to the Hausdorff metric in a hyperspace are also very useful when studying properties of compact metric spaces. For example, Bing [3] proved, using such limits, that two nondegenerate hereditarily indecomposable continua are homeomorphic if each can be chained.

Ingram [7] and Mahavier [7, 9] introduced the concept of inverse limits of inverse sequences of compact Hausdorff spaces with upper semicontinuous multi-valued bonding functions.

The author $[1,2]$ introduced a standard procedure for obtaining inverse sequences of compact metric spaces $X_{n}$ and upper semicontinuous multi-valued bonding functions $\tilde{f}_{n}: X_{n+1} \rightarrow X_{n}$ from one-valued maps $f_{n}$. The procedure defines the graph $\Gamma\left(\tilde{f}_{n}\right)$ as the union of $\Gamma\left(f_{n}\right)$ and the product $A_{n+1} \times X_{n}$, for a closed subset $A_{n+1}$ of $X_{n+1}$. He obtained results on dimension of inverse limits of $\left\{X_{n}, \tilde{f}_{n}\right\}_{n=1}^{\infty}$ and applied it to the problem of finding nontrivial examples of continua, in which given inverse limits are convergence continua.

In this article we show, using ideas from [1, 2], that the inverse limit of any inverse sequence of compact metric spaces and surjective bonding maps is in fact the limit of a sequence of homeomorphic copies of the same spaces in an appropriate hyperspace, with respect to the Hausdorff metric. Our main goal is to prove the following theorem.

Received 26th September, 2006

This work was supported in part by the Slovenian Research Agency, under Grant L2-7207 (C).

Copyright Clearance Centre, Inc. Serial-fee code: 0004-9727/07 \$A2.00+0.00. 
THEOREM 1.1. Let $\left\{X_{n}, f_{n}\right\}_{n=1}^{\infty}$ be an inverse sequence of compact metric spaces $X_{n}$ and surjective maps $f_{n}: X_{n+1} \rightarrow X_{n}$, such that only finitely many spaces are degenerate. Then there is a sequence $\left\{Y_{n}\right\}_{n=1}^{\infty}$ of compact metric spaces in $\prod_{n=1}^{\infty} X_{n}$, such that

(1) for each positive integer $n, Y_{n}$ is homeomorphic to $X_{n}$;

(2) for each positive integer $n, Y_{n} \cap\left(\varliminf_{n}\left\{X_{n}, f_{n}\right\}_{n=1}^{\infty}\right)=\emptyset$;

(3) $\lim _{n \rightarrow \infty} Y_{n}=\lim _{\longleftrightarrow}\left\{X_{n}, f_{n}\right\}_{n=1}^{\infty}$.

\section{Definitions and Notations}

When referring to a space, the term degenerate is synonymous with being a onepoint space, while the term nondegenerate means that the space consists of more than one point.

A map is a continuous function. Let $f$ be a function from $X$ onto $X$, then $f^{2}$ denotes the composition $f \circ f$, and inductively $f^{n}$ denotes the composition $f^{n-1} \circ f$. We use $f^{-n}(Y)$ to denote $\left(f^{n}\right)^{-1}(Y)=\left\{x \in X \mid f^{n}(x) \in Y\right\}$.

Let $\left(X_{1}, d_{1}\right),\left(X_{2}, d_{2}\right),\left(X_{3}, d_{3}\right), \ldots$ be metric spaces with each of the metrics $d_{n}$ bounded by 1 . The metric we use on the product $\prod_{n=1}^{\infty} X_{n}$ is given by

$$
\mathrm{d}\left(\left(x_{1}, x_{2}, x_{3}, \ldots\right),\left(y_{1}, y_{2}, y_{3}, \ldots\right)\right)=\sum_{n=1}^{\infty} \frac{d_{n}\left(x_{n}, y_{n}\right)}{2^{n}} .
$$

Let $(X, d)$ be a compact metric space and $2^{X}$ be the set of all nonempty closed subsets of $X$. We shall use the Hausdorff metric (for details see $[11$, p. 52]) on the hyperspace $2^{X}$ given by

$$
H_{d}(A, B)=\inf \left\{\varepsilon>0 \mid B \subseteq N_{\varepsilon}(A), A \subseteq N_{\varepsilon}(B)\right\} .
$$

For further reading on hyperspaces we recommend $[6,11]$.

Given an inverse sequence $\left\{X_{n}, f_{n}\right\}_{n=1}^{\infty}$ of compact metric spaces $X_{n}$ and maps $f_{n}$ : $X_{n+1} \rightarrow X_{n}$, we define the inverse limit space $\varliminf_{\lfloor}\left\{X_{n}, f_{n}\right\}_{n=1}^{\infty}$ as the subspace of the product $\prod_{n=1}^{\infty} X_{n}$, which consists of all sequences $\left\{x_{n}\right\}_{n=1}^{\infty}$ such that $f_{n}\left(x_{n+1}\right)=x_{n}$ for every positive integer $n$.

Let $X$ and $Y$ be compact metric spaces and let $f: X \rightarrow 2^{Y}$ be a function. The function $f$ is upper semicontinuous at a point $x \in X$ provided that for each open set $V$ in $Y$ containing $f(x)$, there is an open set $U$ in $X$ containing $x$ such that if $y \in U$, then $f(y) \subseteq V$. The function $f$ is upper semicontinuous if it is upper semicontinuous at $x \in X$ for all $x \in X$. The graph $\Gamma(f)$ of $f: X \rightarrow 2^{Y}$ is the set of all points $(x, y) \in X \times Y$ such that $y \in f(x)$. 
For a given inverse sequence $\left\{X_{n}, f_{n}\right\}_{n=1}^{\infty}$, where all $X_{n}$ are compact metric spaces and every $f_{n}$ is an upper semicontinuous function $X_{n+1} \rightarrow 2^{X_{n}}$, the inverse limit $\varliminf_{n}\left\{X_{n}, f_{n}\right\}_{n=1}^{\infty}$ is the subspace of the product $\prod_{n=1}^{\infty} X_{n}$, which consists of all sequences $\left\{x_{n}\right\}_{n=1}^{\infty}$ such that $x_{n} \in f_{n}\left(x_{n+1}\right)$ for every positive integer $n$.

We shall also say that a function $f: X \rightarrow 2^{Y}$ is a multi-valued function from $X$ to $Y$, while writing $f: X \rightarrow Y$.

Let $X$ and $Y$ be compact metric spaces and $f: X \rightarrow Y$ a map. For a closed subset $A \subseteq X$ we define the multi-valued function $\tilde{f}: X \rightarrow Y$ as

$$
\tilde{f}(x)=\left\{\begin{array}{cc}
\{f(x)\} ; & x \notin A \\
Y ; & x \in A .
\end{array}\right.
$$

It was proved in [2], that $\tilde{f}$ is an upper semicontinuous set-valued function from $X$ to $Y$.

Let $\left\{X_{n}, f_{n}\right\}_{n=1}^{\infty}$ be an inverse sequence of compact metric spaces $X_{n}$ and maps $f_{n}$ : $X_{n+1} \rightarrow X_{n}$, and let for each $n=1,2,3, \ldots, A_{n}$ be a closed subset of $X_{n}$. Then we define $D_{n}$ as the subspace of the product $\prod_{i=n}^{\infty} X_{i}$, consisting of all points $\bar{x}, \bar{x}=\left(x_{1}, x_{2}, x_{3}, \ldots\right)$, such that

(1) $x_{1} \in A_{n}$;

(2) for each $i=1,2,3, \ldots, x_{i} \in \tilde{f}_{i+n-1}\left(x_{i+1}\right)$.

We call $D_{n}$ the $n$-tree for sequences $\left\{X_{n}\right\}_{n=1}^{\infty},\left\{A_{n}\right\}_{n=1}^{\infty}$ and $\left\{f_{n}\right\}_{n=1}^{\infty}$.

Let for each $n=1,2,3, \ldots, X_{n}$ be a compact metric space, $f_{n}$ a map from $X_{n+1}$ to $X_{n}$, and $A_{n}$ a closed subset of $X_{n}$. For all $m=1,2, \ldots$ and each $\bar{a} \in D_{m+2}$ we define $L(\bar{a}, m)$ as follows.

$$
L(\bar{a}, m)=\left\{\left(f_{1}\left(f_{2}\left(\ldots f_{m}(x) \ldots\right)\right), \ldots, f_{m-1}\left(f_{m}(x)\right), f_{m}(x), x, \bar{a}\right) \mid x \in X_{m+1}\right\},
$$

and

$$
L(\bar{a}, 0)=\left\{(x, \bar{a}) \mid x \in X_{1}\right\} .
$$

We shall use the following theorem.

Theorem 2.1. ([2]) For all $m \geqslant 0$ and all $\bar{a} \in D_{m+2}, L(\bar{a}, m)$ is homeomorphic to $X_{m+1}$.

\section{INVERSE LIMITS AS LIMITS WITH RESPECT TO THE HAUSDORFF METRIC}

In this section we prove Theorem 1.1. Before proving it, let us prove the following easy lemma, that will be used to prove Theorem 1.1.

LEMMA 3.1. Let $\left\{X_{n}, f_{n}\right\}_{n=1}^{\infty}$ be an inverse sequence of compact metric spaces $X_{n}$, such that only finitely many spaces are degenerate, and arbitrary maps $f_{n}: X_{n+1}$ 
$\rightarrow X_{n}$. Then there exists a point $\bar{x}=\left(x_{1}, x_{2}, x_{3}, \ldots\right) \in \prod_{n=1}^{\infty} X_{n}$, such that $x_{n} \neq f_{n}\left(x_{n+1}\right)$ for infinitely many integers $n$.

PROOF: Let $k$ be the greatest integer, such that $X_{k}$ is degenerate.

CASE 1. There is an integer $n_{0}>k$, such that for all $n \geqslant n_{0}, f_{n}$ is a constant map. For each $n \geqslant n_{0}$, let $y_{n} \in X_{n}$ be such that for each $x \in X_{n+1}, f_{n}(x)=y_{n}$. For $x_{n}, n=1,2,3, \ldots, n_{0}-1$, we may choose any element from $X_{n}$. For $n \geqslant n_{0}$, let $x_{n} \in X_{n} \backslash\left\{y_{n}\right\}$. Obviously, for $\bar{x}=\left(x_{1}, x_{2}, x_{3}, \ldots\right), \bar{x} \in \prod_{n=1}^{\infty} X_{n}$, and $x_{n} \neq f_{n}\left(x_{n+1}\right)$ for infinitely many integers $n$.

CASE 2. For each integer $n_{0}>0$, there is an integer $n>n_{0}$, such that $f_{n}$ is not a constant map. Let $n_{1}, n_{2}, \ldots$ be the increasing sequence of all positive integers such that $f_{n_{k}}$ is not constant. First we take an element $x_{1} \in X_{1}$. For $n>1$ we proceed as follows. If $n \notin\left\{n_{\ell}+1 \mid \ell=1,2,3, \ldots\right\}$ we take for $x_{n}$ any element from $X_{n}$. If $n \in\left\{n_{\ell}+1 \mid \ell=1,2,3, \ldots\right\}$. Clearly, for $\bar{x}=\left(x_{1}, x_{2}, x_{3}, \ldots\right), \bar{x} \in \prod_{n=1}^{\infty} X_{n}$, and $x_{n} \neq f_{n}\left(x_{n+1}\right)$ for infinitely many integers $n$.

Now let us prove Theorem 1.1.

Proof: By Lemma 3.1 we choose and fix

$$
\bar{x}=\left(x_{1}, x_{2}, x_{3}, \ldots\right) \in \prod_{n=1}^{\infty} X_{n},
$$

such that $x_{n} \neq f_{n}\left(x_{n+1}\right)$, for infinitely many integers $n$. For each positive integer $n$, let $\overline{x_{n}}=\left(x_{n}, x_{n+1}, x_{n+2}, \ldots\right) \in \prod_{i=n}^{\infty} X_{i}$. We shall construct the spaces $Y_{n}$ in $\prod_{n=1}^{\infty} X_{n}$, using the upper semicontinuous functions $\tilde{f}_{n}$ for $A_{n}=\left\{x_{n}\right\}$. Let for each $n, D_{n}$ be the $n$ tree for sequences $\left\{X_{n}\right\}_{n=1}^{\infty},\left\{A_{n}\right\}_{n=1}^{\infty}$ and $\left\{f_{n}\right\}_{n=1}^{\infty}$. Hence $\overline{x_{n}} \in D_{n}$ for all $n$. For each positive integer $n$, define $Y_{n}=L\left(\overline{x_{n+1}}, n-1\right)$. By Theorem $2.1, Y_{n}$ is homeomorphic to $X_{n}$ for each $n$. As $x_{m} \neq f_{m}\left(x_{m+1}\right)$ for infinitely many integers $m$, it follows that $Y_{n} \cap\left(\lim _{\longleftarrow}\left\{X_{n}, f_{n}\right\}_{n=1}^{\infty}\right)=\emptyset$ for all $n$. To complete the proof, we only need to show that $\lim _{n \rightarrow \infty} Y_{n}=\lim _{n}\left\{X_{n}, f_{n}\right\}_{n=1}^{\infty}$. We shall prove that by showing that for each $\varepsilon>0$, there is a positive integer $n_{0}$ such that

$$
H_{d}\left(Y_{n}, \lim _{\longleftarrow}\left\{X_{n}, f_{n}\right\}_{n=1}^{\infty}\right)<\varepsilon
$$

for all $n>n_{0}$. Take $\varepsilon>0,0<\varepsilon^{\prime}<\varepsilon$ and a positive integer $n_{0}$ such that

$$
\sum_{i=1}^{\infty} \frac{1}{2^{n_{0}+i}}<\varepsilon^{\prime}
$$

Let $n>n_{0}$. First we show that for each $y \in Y_{n}$, there is $z \in \varliminf^{\lim }\left\{X_{n}, f_{n}\right\}_{n=1}^{\infty}$ such that $d(y, z) \leqslant \varepsilon^{\prime}$. Take $y=\left(y_{1}, y_{2}, y_{3}, \ldots, y_{n-1}, y_{n}, y_{n+1}, \ldots\right) \in Y_{n}$ and let 
$z=\left(y_{1}, y_{2}, y_{3}, \ldots, y_{n-1}, y_{n}, z_{n+1}, z_{n+2}, \ldots\right)$, where $z_{n+1} \in f_{n}^{-1}\left(\left\{y_{n}\right\}\right)$ and for $i=n+2, n+3, \ldots, z_{i} \in f_{i-1}^{-1}\left(\left\{z_{i-1}\right\}\right)$ (recall that all the bonding maps are surjective, so all $z_{i}$ may be chosen). Clearly

$$
d(y, z) \leqslant \sum_{i=1}^{\infty} \frac{1}{2^{n_{0}+i}} \leqslant \varepsilon^{\prime}<\varepsilon .
$$

Now we show that for each $y \in \varliminf_{n}\left\{X_{n}, f_{n}\right\}_{n=1}^{\infty}$, there is $z \in Y_{n}$ such that $d(y, z) \leqslant \varepsilon^{\prime}$. Take $y=\left(y_{1}, y_{2}, y_{3}, \ldots, y_{n-1}, y_{n}, y_{n+1}, \ldots\right) \in \varliminf_{\lfloor}\left\{X_{n}, f_{n}\right\}_{n=1}^{\infty}$. Let

$$
z=\left(y_{1}, y_{2}, \ldots, y_{n-1}, y_{n}, x_{n+1}, x_{n+2}, \ldots\right) \text {, }
$$

where $\left(x_{n+1}, x_{n}, \ldots\right)=\overline{x_{n+1}}$. Clearly $z \in Y_{n}$, and $d(y, z) \leqslant \varepsilon^{\prime}<\varepsilon$. Therefore

$$
H_{d}\left(Y_{n}, \varliminf_{n}\left\{X_{n}, f_{n}\right\}_{n=1}^{\infty}\right) \leqslant \varepsilon^{\prime}<\varepsilon .
$$

REMARK 3.2. If infinitely many spaces $X_{n}$ were degenerate, then, as all the bonding maps are surjective, all the spaces would be degenerate. In this case the inverse limit $\varliminf_{\mathfrak{m}}\left\{X_{n}, f_{n}\right\}_{n=1}^{\infty}$ equals to the product $\prod_{n=1}^{\infty} X_{n}$, which is also a degenerate space.

\section{AN application in CONTINUUM theORY}

In this section we give an application of Theorem 1.1 in continuum theory, precisely, to $\mathcal{P}$-like continua. First look at the basic definitions.

A continuum is a nonempty, compact and connected metric space.

Let $X$ and $Y$ be metric spaces. A map $f: X \rightarrow Y$ is an $\varepsilon$-map if the set $f^{-1}(\{f(x)\})$ has the diameter less than $\varepsilon$ for all $x \in X$. We say that for a family of connected polyhedra $\mathcal{P}$, a continuum $K$ is $\mathcal{P}$-like, if for every $\varepsilon>0$ there is a surjective $\varepsilon$-map $f: K \rightarrow P$ for a continuum $P \in \mathcal{P}$.

THEOREM 4.1. Let $\mathcal{P}$ be a nonempty collection of connected polyhedra and $K$ a nondegenerate $\mathcal{P}$-like continuum. Then there is an inverse sequence $\left\{L_{n}, f_{n}\right\}_{n=1}^{\infty}$ such that for each $n, L_{n} \in \mathcal{P}$ and $f_{n}: L_{n+1} \rightarrow L_{n}$ is a surjective map, and a sequence $\left\{Y_{n}\right\}_{n=1}^{\infty}$ of subspaces of $\prod_{n=1}^{\infty} L_{n}$, such that

(1) $K$ is homeomorphic to $\varliminf_{\mathfrak{i m}}\left\{L_{n}, f_{n}\right\}_{n=1}^{\infty}$;

(2) for each positive integer $n, Y_{n}$ is homeomorphic to $L_{n}$;

(3) for each positive integer $n, Y_{n} \cap\left(\varliminf_{n}\left\{L_{n}, f_{n}\right\}_{n=1}^{\infty}\right)=\emptyset$;

(4) $\lim _{n \rightarrow \infty} Y_{n}=\varliminf_{\lfloor}\left\{L_{n}, f_{n}\right\}_{n=1}^{\infty}$.

Proof: It is well known that every $\mathcal{P}$-like continuum can be represented as an inverse limit of an inverse sequence of elements $L_{n} \in \mathcal{P}$ and surjective bonding maps $f_{n}: L_{n+1} \rightarrow L_{n}$ (for details see [10, p. 148], [11, p. 247]). So $K$ is homeomorphic to an 
inverse limit $\varliminf_{\mathfrak{m}}\left\{L_{n}, f_{n}\right\}_{n=1}^{\infty}$, where for each $n, L_{n} \in \mathcal{P}$ and $f_{n}: L_{n+1} \rightarrow L_{n}$ is a surjective map. Hence, by Theorem 1.1, there is a sequence of continua $Y_{n} \subseteq \prod_{k=1}^{\infty} L_{k}$, such that for each $n, Y_{n}$ is homeomorphic to $L_{n}$, and $\varliminf_{\lfloor}\left\{L_{n}, f_{n}\right\}_{n=1}^{\infty} \cap Y_{n}=\emptyset$.

It is a well known fact that every continuum can be arbitrarily approximated by arcs with respect to the Hausdorff metric. Let $X=[0,1] \times[0,1]$. It is easy to construct a sequence of arcs in plane $\left\{L_{n}\right\}_{n=1}^{\infty}$ such that $\lim _{n \rightarrow \infty} L_{n}=X$ with respect to the Hausdorff metric. Obviously $X$ is not arclike ( $X$ contains a triod $[11$, p. 231]) but every inverse limit of arcs and surjective bonding maps is arclike (for details see [11, p. 246]). Therefore there are limits $\lim _{n \rightarrow \infty} X_{n}$ of compact metric spaces with respect to the Hausdorff metric that are not homeomorphic to any of inverse limits of homeomorphic copies of $X_{n}$ and surjective bonding maps.

\section{REFERENCES}

[1] I. Banič, 'Continua with kernels', Houston J. Math. (2006) (to appear).

[2] I. Banič, 'On dimension of inverse limits with upper semicontinuous set-valued bonding functions', (submitted).

[3] R.H. Bing, 'Concerning hereditarily indecomposable continua', Pacific J. Math. 1 (1951), 43-51.

[4] L. Block, J.E. Keesling and V.V. Uspenskij, 'Inverse limits which are the pseudoarc', Houston J. Math. 26 (2000), 629-638.

[5] G.W. Henderson, 'The pseudo-arc as an inverse limit with one bonding map', Duke Math. J. 31 (1964), 421-425.

[6] A. Illanes and S.B. Nadler, Hyperspaces: fundamentals and recent advances (M. Dekker, New York, 1999).

[7] W.T. Ingram and W.S. Mahavier, 'Inverse limits of upper semicontinuous set valued functions', Houston J. Math. 32 (2006), 119-130.

[8] K. Kuratowski, Topology, Vol. 2 (Academic Press and PWN, New York, London and Warszawa, 1968).

[9] W.S. Mahavier, 'Inverse limits with subsets of [0,1] $\times[0,1]$ ', Topology Appl. 141 (2004), 225-231.

[10] S. Mardešić and J. Segal, ' $\epsilon$-Mappings onto polyhedra', Trans. Amer. Math. Soc. 109 (1963), 146-164.

[11] S.B. Nadler, Continuum theory: an introduction (Marcel Dekker, New York, 1992).

Department of Mathematics

Faculty of Mechanical Engineering

University of Maribor

Smetanova 17, Maribor 2000

Slovenia

e-mail: iztok.banic@uni-mb.si 